\begin{tabular}{ccc|} 
PEDIOMATERNAL \\
NURSING JOURNAL \\
Vol. 7, No. 2, September 2021 \\
Journal Homepage: https://e-journal.unair.ac.id/PMNJ/ \\
http://dx.doi.org/10.20473/pmnj.v7i2.27497
\end{tabular}

Review

\title{
A Systematic Review of Endocrine Therapy for Improved Reproductive and Metabolic Outcomes in PCOS Women
}

\author{
Nur Aini Lutfi Rahmawati ${ }^{1^{*}} \mathbb{D}$, Esti Yunitasari ${ }^{1}{ }^{\mathbb{D}}$, Ni Ketut Alit Armini ${ }^{1}{ }^{\mathbb{D}}$, Padoli \\ Padoli $\left.^{2}{ }^{(}\right)$, Suharyono Suharyono $^{1}$ (D) \\ ${ }^{1}$ Faculty of Nursing, Universitas Airlangga, Surabaya, Indonesia \\ ${ }^{2}$ Department of Nursing, Poltekkes Kemenkes Surabaya, Surabaya, Indonesia
}

\section{ARTICLE HISTORY}

Received : June 14, 2021

Revised : July 10, 2021

Accepted : July 21, 2021

Published : August 30, 2021

\section{KEYWORDS}

endocrine therapy; polycystic ovary syndrome; reproductive outcomes; metabolic outcomes

\section{CORRESPONDING AUTHOR}

*Nur Aini Lutfi Rahmawati nur.aini.lutfi-2019@fkp.unair. ac.id

Faculty of Nursing, Universitas Airlangga, Surabaya, Indonesia

Cite this as:

\section{INTRODUCTION}

Polycystic ovarian syndrome (PCOS) is a prevalent endocrine condition that affects $5-10 \%$ of reproductive-age women. Chronic anovulation results in menstrual dysregulation (oligomenorrhea, amenorrhea) and increased

\begin{abstract}
Introduction: Polycystic ovarian syndrome (PCOS) is characterized by infrequent or absent ovulation as well as elevated levels of androgens and insulin (hyperinsulinemia). The purpose of this study was to determine the efficacy of endocrine treatment in improving reproductive and metabolic outcomes in women with PCOS.
\end{abstract}

Methods: We searched the following databases from inception to March 2020: PubMed, ProQuest, ScienceDirect, Scopus and CINAHL. We investigated at metformin, clomiphene citrate, metformin plus clomiphene citrate, D-chiro-inositol, statins, and resveratrol as treatments. We compared them to each other, as well as to a placebo or no therapy. The quality of the evidence ranged from extremely low to moderate. The risks of bias (poor reporting of technique and inadequate outcome data), imprecision, and inconsistency were the limitations.

Results: Although the evidence quality was low, our latest evaluation indicated that metformin alone may be superior to placebo for live birth. Data for live birth were equivocal when metformin was compared to clomiphene citrate, and our conclusions were hampered by a paucity of evidence. Body mass index (BMI) varies in the results, emphasizing the need of stratifying data by BMI.

Conclusion: Clinical pregnancy and ovulation improvements demonstrate that clomiphene citrate is still preferred to metformin for ovulation induction in obese women with PCOS.

Rahmawati, N, A, L., Yunitasari, E., Armini, N, K, A., Padoli, P., Suharyono, S. (2021). A Systematic Review of Endocrine Therapy for Improved Reproductive and Metabolic Outcomes in PCOS Women. Pediomaternal Nurs. J., 7(2), 65-76. Doi: http://dx.doi.org/10.20473/pmnj.v7i2.27497 
ethnic groups (ranging from 2.2 to 26 percent) (Jensterle et al., 2020). Chronic anovulation with or without menstrual cycle irregularities, clinical or biochemical hyperandrogenism, and ultrasound confirmation of morphologically polycystic ovaries are the major features of the condition (Macut, Božić-Antić, et al., 2017). The most common sign of hyper androgenization is hirsutism. Other skin-related symptoms including acne, seborrhea, androgenic alopecia are uncommon in PCOS patients (Cozzolino et al., 2020).

In terms of reproduction, this condition impacts fertility in women of reproductive age and determines the possibility of postmenopausal neoplastic changes. The metabolic part of the syndrome has a substantial impact on the women in question's whole lives, primarily because it can alter over time, growing worse in certain situations (Macut, Božić-Antić, et al., 2017). Furthermore, the majority of women with PCOS have metabolic problems such as insulin resistance, obesity, and dyslipidemia. Insulin resistance is seen in both obese and non-obese women with PCOS (Morgante et al., 2020).

Women with PCOS have high plasma concentrations of ovarian and adrenal androgens, gonadotropin abnormalities, a relative increase in estrogen levels (especially estrone) derived from peripheral androgen conversion, low serum levels of sex hormonebinding globulin (SHBG), and often high serum insulin levels (INS) (Almalki et al., 2020). According to the study from Moradi etal.,(2020), The presence of excess androgens, primarily of ovarian origin, is a major characteristic of the condition. However, an adrenal source should always be examined. Most, but not all, women with PCOS have elevated androgen levels in their blood. Androstenedione (A) and testosterone (T) are ovarian androgen secretion indicators, whereas dehydroepiandrosterone sulfate (DHEAS) is the best adrenal secretion marker (Dokras et al., 2017). As PCOS dramatically as a result and worsen, they can frequently lead to a major decline in quality of life, increased stress, and a poor impact on psychological and sexual well-being (Della Corte et al., 2020). Women with PCOS are at a higher risk of comorbidities and long-term health repercussions. These women are more likely to acquire problems such as type II diabetes, obesity, hypertension, dyslipidemia, and cardiovascular disease (Podfigurna et al., 2020). Zhang et al. observed considerably greater serum irisin levels in PCOS women than in healthy women. Circulating irisin levels were linked to hyperandrogenism but not oligoanovulation or PCO morphology (B. Zhang et al., 2020).

Insulin resistance is a symptom of PCOS. It is more common in obese women, indicating that PCOS and obesity work together to increase the severity of insulin dysregulation. Obesity increases beta-cell insulin production and causes compensatory hyperinsulinemia. Insulin resistance, which is caused by hyperinsulinemia, has been related to all of the symptoms of PCOS, including hyperandrogenism, reproductive abnormalities, acne, hirsutism, and metabolic problems (Cozzolino et al., 2020). Multiple processes are considered to contribute to hyperandrogenism, including insulin resistance and compensatory hyperinsulinemia. Hyperinsulinemia promotes ovarian androgen production both directly and indirectly by acting on thecal cells and blocking FSH effect on granulosa cells. Insulin enhances thecal cell proliferation in vitro, causes androgen production via $\mathrm{LH}$, and enhances cytochrome P450 production of LH and IGF-1 receptors (Charifson \& Trumble, 2019). Furthermore, insulin receptors found in the hypothalamus and pituitary gland increase the release of FSH and LH both at rest and following GnRH activation (Morgante et al., 2020).

Furthermore, insulin impacts hyperandrogenism by inhibiting SHBG production, resulting in increased testosterone availability. In women with PCOS, the IGF-1/ IGFBP-1 ratio rises considerably, making IGF1 more easily accessible to thecal cells and increasing androgen synthesis (Morgante et al., 2020). Furthermore, IGF-1 promotes estrogen synthesis by granulosa cells and can operate synergistically with FSH and LH to modulate aromatase expression in these cells. Insulin stimulates ACTH-mediated androgen synthesis in the adrenal glands, positively influencing LHstimulated ovarian steroidogenesis (Brennan et al., 2017).

Women with PCOS had higher blood pressure, endothelialdysfunction, lowerarterial compliance, central obesity, dyslipidemia, low-grade chronic inflammation, and higher 


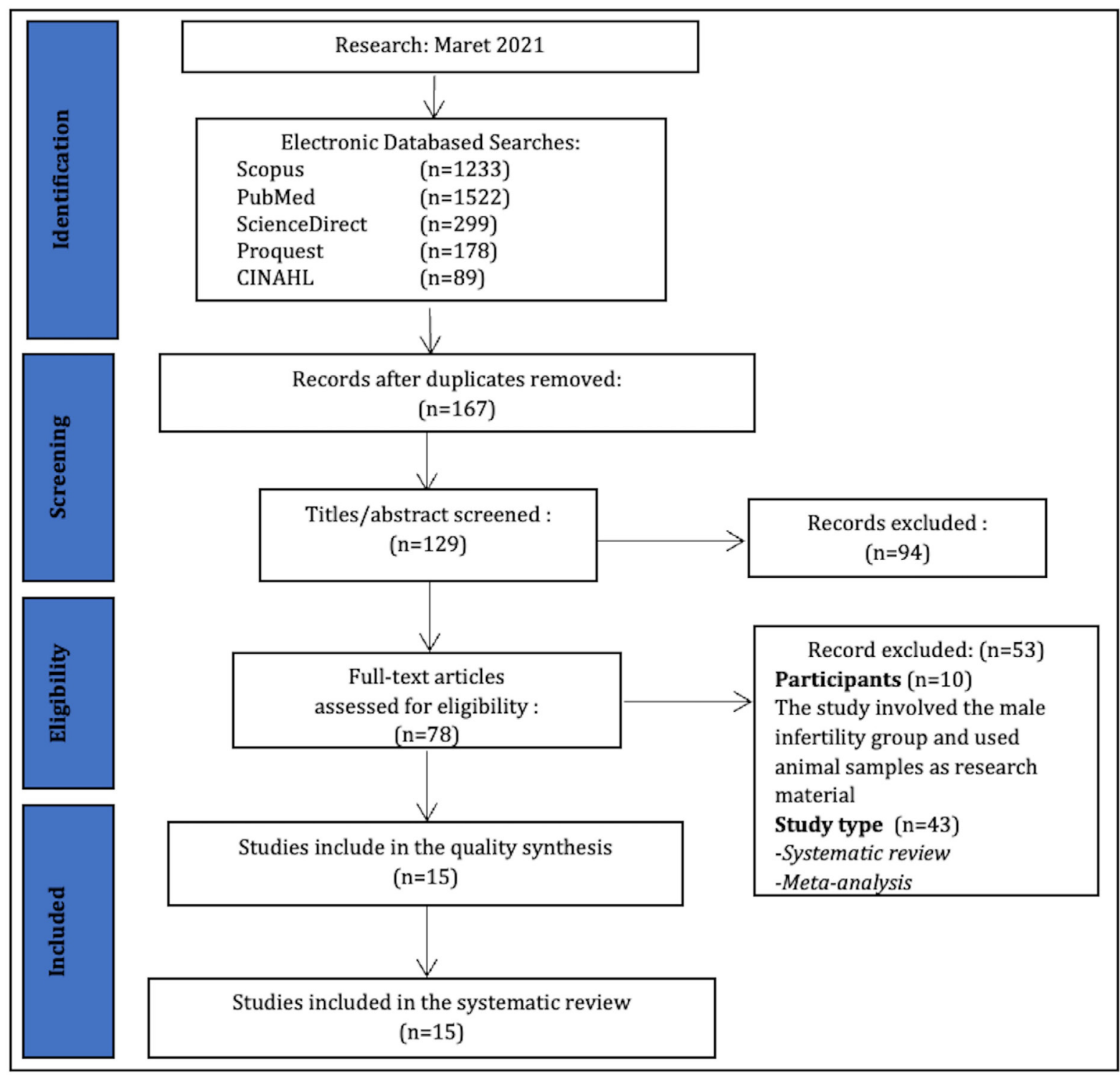

Figure 1. Flow diagram of the study selection, adaptation from Moher 2014

endothelin-1 and homocysteine levels (Altinok et al., 2018). The therapy of PCOS tries to minimize the clinical aspects of the illness and is based mostly on the patient's most common symptoms. Response to treatment is gradual, with biochemical reversal occurring up to 6-9 months before clinical improvement (Sharpe et al., 2019). Treatment of PCOS necessitates a multifaceted approach; non-pharmacological therapies are universally suggested, including adequate food and exercise, since weight loss is necessary if obese or necessary to reestablish insulin sensitivity (Moran et al., 2020). Enhancing insulin sensitivity in women with PCOS by both lifestyle and pharmaceutical intervention has been proposed to alleviate hyperandrogenism, restore ovulation, and increase pregnancy rates. Metformin, the most extensively researched insulin-sensitizing medication in PCOS patients, is frequently linked with a considerable drop in plasma concentrations of different androgens (Q. Y. Wang et al., 2016). The contraceptive pill, which contains estrogens and progestogens, is the most widely used and well-proven means of treating women with PCOS. The contraceptive pill regulates the menstrual cycle in this group of women by blocking the hypothalamicpituitary-ovarian axis. This sort of therapy also has a good impact on symptoms and hormonal markers (De Diego et al., 2020). The most severe impact of the contraceptive pill appears to be an inhibition of testosterone production by the ovaries. Serum $25(\mathrm{OH})$ vitamin D levels 
are linked to Sex-Hormone-Binding Globulin (SHBG), which can be utilized to diagnose PCOS metabolism or endocrine abnormalities (Dokras et al., 2017). This research examined at how endocrine medication affected reproductive and metabolic outcomes in women with PCOS.

\section{METHOD}

\section{Design}

Fifteen studies were included in this systematic review. Scopus, PubMed, Proquest, CINAHL, and ScienceDirect were used as online databases for the primary search strategy to identify articles. The complete search strategy was adapted using terms and Medical Subject Headings (MeSH) combined with Boolean operators. The keywords used to search those journals were "Endocrine" OR "Endocrine System" OR "Endocrine Glands" OR "Chromaffin System" OR "Neurosecretory Systems" AND "Reproduction" OR "Reproduction, Asexual" OR "Reproductive Behavior" AND "Metabolic" OR "Metabolism" OR "Insulin Secretion" OR "Metabolic Networks and Pathways" AND
"Polycystic Ovary Syndrome" OR "Polycystic Ovarian Disease" OR "PCOS".

\section{Instruments}

The feasibility of the study was assessed using the PICOT (Population, Intervention, Comparison, Outcome, Time) framework. The inclusion criteria were (i) patient diagnosed with Polycystic Ovary Syndrome (ii) patient who had symptoms such as diagnosed with PCOS with the following criteria: PCOS according to Rotterdam criteria, appearance of polycystic ovaries on ultrasound, BMI $\geq 25$ $\mathrm{kg} / \mathrm{m} 2$, age 18-40 years (iii) types of study: randomized controlled trials (RCT), casecontrol study, quasy experiment, only articles published in English were included (iv) publication years; 2016 until $2020(\mathrm{v})$ primary outcome: to examine at endocrine treatment as a means of enhancing reproductive and metabolic outcomes in women with PCOS.

\section{Data Extraction}

The following information was extracted from 15 articles: information on demographics, study design, outcome measure, sample size,

Table 1. Study Assessment Results for Systematic Review Using JBI Critical Appraisal Tools

\begin{tabular}{|c|c|c|c|c|c|c|c|c|c|c|c|c|c|c|c|}
\hline \multirow{2}{*}{ No } & \multirow{2}{*}{ Citation } & \multicolumn{13}{|c|}{ The Criteria of JBI Critical Appraisal } & \multirow{2}{*}{ Result } \\
\hline & & 1 & 2 & 3 & 4 & 5 & 6 & 7 & 8 & 9 & 10 & 11 & 12 & 13 & \\
\hline \multicolumn{16}{|c|}{ Randomized Control Trials (7 criteria) } \\
\hline 1 & (J. Zhang et al., 2017) & $\sqrt{ }$ & $\sqrt{ }$ & $\sqrt{ }$ & $\sqrt{ }$ & - & - & $\sqrt{ }$ & $\sqrt{ }$ & $\sqrt{ }$ & $\sqrt{ }$ & $\sqrt{ }$ & $\sqrt{ }$ & $\sqrt{ }$ & $11 / 13(84 \%)$ \\
\hline 2 & (Abdalla et al., 2020) & $\sqrt{ }$ & $\sqrt{ }$ & $\sqrt{ }$ & $\sqrt{ }$ & - & - & $\sqrt{ }$ & $\sqrt{ }$ & $\sqrt{ }$ & $\sqrt{ }$ & $\sqrt{ }$ & $\sqrt{ }$ & $\sqrt{ }$ & $11 / 13(84 \%)$ \\
\hline 3 & (Kim et al., 2020) & $\sqrt{ }$ & $\sqrt{ }$ & $\sqrt{ }$ & - & $\sqrt{ }$ & $\sqrt{ }$ & $\sqrt{ }$ & $\sqrt{ }$ & $\sqrt{ }$ & $\sqrt{ }$ & - & - & $\sqrt{ }$ & $10 / 13(76 \%)$ \\
\hline 4 & (Jensterle et al., 2020) & $\sqrt{ }$ & $\sqrt{ }$ & $\sqrt{ }$ & $\sqrt{ }$ & $\sqrt{ }$ & - & $\sqrt{ }$ & $\sqrt{ }$ & $\sqrt{ }$ & $\sqrt{ }$ & - & $\sqrt{ }$ & $\sqrt{ }$ & $11 / 13(84 \%)$ \\
\hline 5 & (De Diego et al., 2020) & $\sqrt{ }$ & $\sqrt{ }$ & $\sqrt{ }$ & $\sqrt{ }$ & $\sqrt{ }$ & - & - & $\sqrt{ }$ & $\sqrt{ }$ & $\sqrt{ }$ & - & $\sqrt{ }$ & - & $9 / 13(69 \%)$ \\
\hline 6 & (Dokras et al., 2017) & $\sqrt{ }$ & $\sqrt{ }$ & $\sqrt{ }$ & $\sqrt{ }$ & $\sqrt{ }$ & $\sqrt{ }$ & - & $\sqrt{ }$ & $\sqrt{ }$ & $\sqrt{ }$ & $\sqrt{ }$ & $\sqrt{ }$ & $\sqrt{ }$ & $12 / 13(92 \%)$ \\
\hline 7 & $\begin{array}{l}\text { (Macut, Božić-Antić, et al., } \\
\text { 2017) }\end{array}$ & $\sqrt{ }$ & $\sqrt{ }$ & $\sqrt{ }$ & - & $\sqrt{ }$ & $\sqrt{ }$ & $\sqrt{ }$ & $\sqrt{ }$ & $\sqrt{ }$ & - & $\sqrt{ }$ & - & $\sqrt{ }$ & $10 / 13(76 \%)$ \\
\hline \multicolumn{16}{|c|}{ Experimental study (8 criteria) } \\
\hline 8 & (Podfigurna et al., 2020) & $\sqrt{ }$ & $\sqrt{ }$ & $\sqrt{ }$ & $\sqrt{ }$ & $\sqrt{ }$ & $\sqrt{ }$ & $\sqrt{ }$ & $\sqrt{ }$ & $\sqrt{ }$ & & & & & $9 / 9(100 \%)$ \\
\hline 9 & (Altinok et al., 2018) & $\sqrt{ }$ & $\sqrt{ }$ & $\sqrt{ }$ & - & $\sqrt{ }$ & $\sqrt{ }$ & $\sqrt{ }$ & $\sqrt{ }$ & $\sqrt{ }$ & & & & & $8 / 9(88 \%)$ \\
\hline 10 & (Sharpe et al., 2019) & $\sqrt{ }$ & $\sqrt{ }$ & $\sqrt{ }$ & $\sqrt{ }$ & $\sqrt{ }$ & $\sqrt{ }$ & 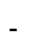 & - & $\sqrt{ }$ & & & & & $7 / 9(77 \%)$ \\
\hline 11 & (Moran et al., 2020) & $\sqrt{ }$ & $\sqrt{ }$ & $\sqrt{ }$ & $\sqrt{ }$ & $\sqrt{ }$ & $\sqrt{ }$ & $\sqrt{ }$ & $\sqrt{ }$ & - & & & & & $8 / 9(88 \%)$ \\
\hline 12 & (Q. Y. Wang et al., 2016) & $\sqrt{ }$ & $\sqrt{ }$ & $\sqrt{ }$ & $\sqrt{ }$ & $\sqrt{ }$ & $\sqrt{ }$ & $\sqrt{ }$ & - & $\sqrt{ }$ & & & & & $8 / 9(88 \%)$ \\
\hline 13 & $\begin{array}{l}\text { (Banaszewska et al., } \\
\text { 2019) }\end{array}$ & $\sqrt{ }$ & $\sqrt{ }$ & $\sqrt{ }$ & $\sqrt{ }$ & $\sqrt{ }$ & $\sqrt{ }$ & $\sqrt{ }$ & $\sqrt{ }$ & $\sqrt{ }$ & & & & & $9 / 9(100 \%)$ \\
\hline 14 & $\begin{array}{l}\text { (Macut, Bjekić-Macut, et } \\
\text { al., 2017) }\end{array}$ & $\sqrt{ }$ & $\sqrt{ }$ & $\sqrt{ }$ & $\sqrt{ }$ & $\sqrt{ }$ & $\sqrt{ }$ & $\sqrt{ }$ & - & - & & & & & $7 / 9(77 \%)$ \\
\hline 15 & (Zeng et al., 2020) & $\sqrt{ }$ & $\sqrt{ }$ & $\sqrt{ }$ & $\sqrt{ }$ & $\sqrt{ }$ & $\sqrt{ }$ & $\sqrt{ }$ & $\sqrt{ }$ & $\sqrt{ }$ & & & & & $9 / 9(100 \%)$ \\
\hline
\end{tabular}


intervention, control, pre-post intervention means, country and year of publication from each study. Information was collected on relevant outcome data and included the number of participants. This systematic review adhered to the recommended reporting elements for systematic reviews (the JBI Critical Appraisal) guidelines for the stages of design and results.

\section{Risk of Bias}

The risk of bias was measured using The JBI Critical Appraisal to assess the quality of each study. Studies with RCT research designs were assessed using the JBI Critical Appraisal Checklist. The checklist consists of some questions with answers to "yes", "no", "unclear", and "not valid". An assessment score that reaches a minimum of $50 \%$ then meets the critical appraisal with the cut-off point value agreed upon by the researcher. We did not include studies with scores below 50\% to avoid bias in results and discussion. The JBI scores for each journal in this review $>50 \%$. The assessment results for all articles used the JBI critical assessment tool for experimental study and the Randomized Control Trial (RCT). The Randomized Control Trial design research got a score of $69 \%$ to $92 \%$ with 7 articles, and the experimental study design score was $77 \%$ to $100 \%$ with a total of 8 articles. The results of the study assessments that have been adjusted to the JBI assessment showed different values. This result indicated the quality of each study reviewed. The results of the assessment of each selected study indicated that all studies scored above $50 \%$, which means that they can be used as a reference for systematic reviews (Table 1 ).

\section{RESULT}

Three steps strategy were used to the initial phase of literature search in fifth databases with the specified keywords then obtained several 3321 articles. It consists of 1233 articles from Scopus, 1522 articles from PubMed, 299 articles from ScienceDirect, 179 articles from Proquest, and 89 articles from CINAHL. The second step was reviewing the abstract retrieved for eligible criteria. We excluded some articles with the PICOT framework. At the end of the process, we included fifteen studies consisting of seven randomised controlled trials, and the other was an experimental study.

\section{Population}

Respondents in the study were PCOS patients identified using the Rotterdam criteria, which included at least two of the following symptoms: 1) oligo or anovulation; 2) clinical or biochemical evidence of hyperandrogenism; 3) polycystic ovary by ultrasonography; BMI 25 $\mathrm{kg} / \mathrm{m} 2$, age $18-40$ years. The incidence of PCOS varies by population, as does the link between PCOS and insulin resistance or obesity. These disparities may be due to hereditary factors as well as changes in lifestyle. Based on the Rotterdam Consensus diagnostic criteria, we included women undergoing ovulation induction who had oligo or anovulatory PCOS. Women undergoing In-Vitro Fertilization (IVF) or intracytoplasmic spermatic injection (ICSI) were omitted since this is covered in a separate Cochrane Review.

\section{Intervention}

Metformin, COCs, metformin with clomiphene citrate, D-chiro-inositol, statins, and resveratrol were the therapies used in the included studies. We compared them to each other, as well as to a placebo or no therapy. The quality of the evidence ranged from extremely low to moderate. The risks of bias (poor reporting of technique and inadequate outcome data), imprecision, and inconsistency were the limitations.

\section{DISCUSSION}

PCOS is a prevalent and complicated endocrine illness that affects up to $13 \%$ of women of reproductive age. It is characterized by oligo-ovulation or anovulation, increased androgen levels, and polycystic ovary abnormalities in ultrasound readings (Altinok et al., 2018). Women with PCOS were more likely to experience infertility or menstrual irregularities. They are also more vulnerable to systemic endocrine diseases, which include not just reproductive endocrine problems but also insulin resistance, which can lead to aberrant glucose and lipid metabolism or even cardiocerebrovascular disease, particularly in PCOS patients who are overweight (Podfigurna et al., 2020). The results of a systematic review study showed that several hormonal therapies can be used as a guide in improving the quality of 
Table 2. Related Studies Results ( $\mathrm{n}=15)$

\begin{tabular}{ll}
\hline Author & Outcome \\
\hline (J. Zhang et al., 2017) & $\begin{array}{l}\text { Lifestyle changes combined with metformin and clomiphene have been shown to enhance } \\
\text { the reproductive endocrine and lipid metabolism of obese PCOS patients, reduce the sizes } \\
\text { of the left and right ovaries, and boost menstruation recovery, ovulation, and pregnancy } \\
\text { rates. }\end{array}$
\end{tabular}

(Q. Y. Wang et al., 2016) When compared to CPAcontaining COCs, DRPcontaining COCs paired with metformin and lifestyle changes might better regulate BP and correct carbohydrate metabolism in women with PCOS and metabolic disorders.

(Sharpe et al., 2019) Metformin boosts pregnancy rates and may boost ovulation rates. If metformin and clomiphene citrate (CC) are used together, the combination treatment group had a greater rate of clinical pregnancy and ovulation.

(Altinok et al., 2018) Therapy with M or OCP was preferable to treatment with OCP in terms of lowered FG score, while therapy with MOCP was superior to treatment with OCP in terms of weight reduction.

(Jensterle et al., 2020) Metformin should not be avoided in the treatment of PCOS in overweight-obese women with normal fasting glucose and glucose tolerance, and metformin therapy decisions are based on BMI, oligomenorrhea, and biochemical hyperandrogenism regardless of glycemic status.

(Podfigurna et al., 2020) OCs inhibit LH and FSH release, lowering ovarian androgen synthesis while increasing sex hormone-binding globulin (SHBG) synthesis in the liver.

(Kim et al., 2020) Except for serum testosterone levels, LSM had more benefits than metformin alone. When compared to LSM alone, the effectiveness of LSM + metformin is restricted to fasting serum insulin levels and menstrual periods, and the addition of metformin to LSM resulted in no significant improvements in decreasing BMI.

(Macut, Božić-Antić, et Metformin may improve the unfavourable metabolic profile and restore ovulation in al., 2017) women with PCOS, making it the medicine of choice above other insulin sensitizers used to treat PCOS patients with NAFLD.

(Morgante et al., 2020) There is a link between metformin dosage, BMI, and hyperandrogenism in overweight PCOS women, implying that the greater the BMI index, the higher the dose of metformin required to achieve an effective decrease in insulin resistance in these individuals.

(Banaszewska et al., Metformin, an insulin sensitizer, has the most experience treating PCOS and is indicated as 2019) first-line therapy in the treatment of metabolic/glycemic disorders. Statins are medications that are used in conjunction with other medications to treat hyperandrogenemia, chronic inflammation, and to enhance lipid profiles. Resveratrol is the most recent therapeutic adjuvant medication in PCOS, having potent anti-inflammatory, antioxidant, and cardioprotective properties.

(Macut, Bjekić-Macut, et Insulin resistance and compensatory hyperinsulinemia are thought to be common in al., 2017) PCOS women. The major effects of PCOS are further metabolic derangements and low reproductive outcomes.

(Zeng et al., 2020) PCOS is a diverse illness with unknown pathogenic causes. Excess androgen not only causes PCOS, but it may also combine with other variables such as insulin resistance, hyperinsulinemia, and obesity to worsen the condition.

(Abdalla et al., 2020) For the treatment of metabolic comorbidities in PCOS, a wide range of treatment approaches with potential benefits are available. The combination of lifestyle management, metformin, GLP-1 RA, SGLT-2 inhibitors, and bariatric surgery outperforms monotherapy in terms of metabolic benefits and improvement of metabolic comorbidity markers.

(Almalki et al., 2020) Atorvastatin was found to be more successful than other treatment techniques in lowering total testosterone levels in PCOS patients. The usage of atorvastatin COC, spironolactone plus metformin, simvastatin, spironolactone, simvastatin plus metformin, metformin, lifestyle change, and placebo was linked with the highest decrease in testosterone levels.

(Dokras et al., 2017) Oral combination hormonal contraceptives administered at a modest dose (20 mcg Ethinyl estradiol) for 16 weeks are related with a considerable increase in cholesterol efflux capability (CEC). 
life of PCOS patients, of which 15 articles were found that discussed hormonal (endocrine) therapy and its relationship with PCOS.

A study by Kim, Chon and Lee, (2020b) claimed that PCOS occurs when the ovaries are driven to create an excess of androgens, particularly testosterone, by releasing an excess of LH hormone in the anterior pituitary gland. This study interferes with follicle development. It is this excess LH value that is thought to be the leading cause of PCOS ovarian hypergonadism in the stimulant effect of LH on theca cells. The increase in insulin levels directly contributes to the abnormalities which are seen in the hypothalamus-pituitary-ovary. This condition directly affects PCOS occurrence with hyperinsulinemia mechanisms that increase: the frequency of GnRH (GonadotropinReleasing Hormone) secretion, LH production exceeds FSH, and ovarian androgen production increased, follicular maturation decreased, and sex-hormone-binding globulin (SHBG) binding decreased. These factors contribute to the development of PCOS. A Prior study by Macut, Božić-Antić, et al., (2017) found that the increased steroidogenic potential of theca cells will increase the aromatase enzyme activity (CYP19). It works by encoding cytochrome p450 aromatase in the ovaries, responsible for converting testosterone to estradiol-induced by FSH during early follicular development.

According to Morgante et al., (2020), According to Morgante et al., (2020), various pharmacological therapies have been used in treating PCOS. The therapies include ovulatory dysfunction-related infertility (clomiphene citrate, metformin, aromatase inhibitors, and glucocorticoids), menstrual disorders (cyclic progestins and combination oral contraceptives such as estrogen and progestin), and androgen related symptoms (anti-androgens, glucocorticoids, gonadotropin-releasing hormone agonists, oral contraceptives such as ethinyl estradiol). Following research conducted by Almalki et al., (2020), Ovulation induction medications such as letrozole, clomiphene citrate (with or without metformin), and gonadotropins are first-line pharmacological treatments for anovulatory infertility in women with PCOS. These medications can increase the risk of multiple pregnancies or ovarian hyperstimulation syndrome, which is linked to gastrointestinal problems. Clomiphene citrate (Clomid) is a tablet and is the most commonly used drug to help women ovulate (produce eggs). Zeng et al., (2020) showed that clomiphene citrate has a $60-85 \%$ success rate in helping women produce eggs and has a pregnancy success rate of between $30-50 \%$ after 6 ovulation cycles (6 months). In accordance with research conducted by Jensterle et al., (2020) an increase in ovulation with lifestyle modification through treatment with clomiphene citrate has been reported.

Androgens are steroid hormones, and excess androgen is the most common clinical manifestation of PCOS (Zeng et al., 2020). As a result, it is critical to understand which androgens are synthesized and how they are normally synthesized in women. Androgens are essential female reproductive endocrine system hormones. Testosterone (T), dihydrotestosterone (DHT), androstenedione (A4), dehydroepiandrosterone (DHEA), and dehydroepiandrosterone sulfate are all examples of androgens (DHEAS). DHT and T are all precursors to A4, DHEA, and DHEAS. Only T and DHT can interact directly with the androgen receptor. In women, the ovaries and adrenal glands are the primary sources of androgens (Macut, Bjekić-Macut, et al., 2017), and steroidogenic enzymes regulate androgen synthesis. Consumption of combined oral contraceptive pills (COCPs) is one of the firstline medical treatments recommended for the management of hyperandrogenism and menstrual cycle regulation in PCOS women (Abdalla et al., 2020; Almalki et al., 2020; Banaszewska et al., 2019; Kim et al., 2020; Macut, Bjekić-Macut, et al., 2017; Podfigurna et al., 2020; Zeng et al., 2020). COCPs have an estrogenic effect; there is an increase in the level of Sex Hormone Binding Globulin (SHBG), which functions to reduce testosterone and progestin, inhibits the secretion of Luteinizing Hormone (LH), which results in decreased androgen production, and suppression of 5reductase activity, which reduces $\mathrm{T}$ to DHT conversion. Long-term use of COCP reduces $\mathrm{mFG}$, total testosterone, free testosterone, androstenedione, and DHEAS while increasing SHBG. Metformin can be used in conjunction with COCPs to prevent weight gain and lower the risk of hyperandrogenism Altinok et al., (2018). Metformin combined with clomiphene 
citrate in infertile, anovulatory PCOS women may be beneficial in cases of clomiphene citrate resistance or obesity, resulting in improved ovulation and pregnancy rates (Balen et al., 2016). Sharpe et al., (2019) Metformin has been used as a treatment option for PCOS metabolic disorders such as insulin resistance, hyperinsulinemia, and insulin sensitizing agent for decades, according to research. Metformin lowers insulin levels, which reduces ovarian and adrenal androgen secretion, pituitary LH secretion, and increases SHBG levels.

Metformin therapy reduced the rates of ovarian hyperstimulation syndrome (OHSS) in patients undergoing assisted reproductive technologies in original studies (Banaszewska et al., 2019). However, a randomized controlled trial failed to replicate these findings, demonstrating that a short course of metformin did not reduce OHSS in GnRH antagonist cycles (Morgante et al., 2020). According to a recent review of OHSS prevention strategies, there was at least moderate quality evidence to consider metformin treatment before and during an ART cycle for women with PCOS (Dokras et al., 2017). Furthermore, preliminary and limited evidence suggested that metformin may increase live birth rates in subfertile PCOS women undergoing gonadotrophin-induced ovulation induction followed by timed intercourse or intrauterine insemination (Abdalla et al., 2020). Another significant problem is metformin treatment in adolescent PCOS women. When adolescent girls are diagnosed with persistent irregular cycles, 2-3 years past menarche, and clinical signs of hyperandrogenemia, metformin is recommended as the first monotherapy in daily doses ranging from $850 \mathrm{mg}$ (lean girls) to $1500-2500 \mathrm{mg}$ per day in overweight young women (Li et al., 2020).

There are many benefits to metformin for PCOS patients, including improving several factors such as BMI, menstrual frequency, fasting glucose levels, fasting insulin levels, testosterone, triglyceride levels, and LH levels (Altinok et al., 2018; Sharpe et al., 2019; Q. Y. Wang et al., 2016; J. Zhang et al., 2017). Research conducted by Kim, Chon and Lee, (2020b) The researchers compared the effects of clomiphene alone versus clomiphene (50 $\mathrm{mg} /$ day) and metformin (2000 mg/day) on ovulation induction and pregnancy in women with PCOS. The combined group had a higher percentage of ovulation and pregnancy than the clomiphene alone group, indicating a significantly higher percentage of live births (22.5 percent vs 7.2 percent ). As a result, metformin can be used as a supplement to, but not as a replacement for, a healthy lifestyle. Metformin may have an additional effect on increasing cardiometabolic risk when combined with lifestyle interventions, particularly in the high metabolic risk group (Sharpe et al., 2019). Metformin, in our opinion, is well established in PCOS therapy and should not be used for all hyperandrogenic women, but only in cases of insulin resistance, glucose intolerance, and a high risk of developing metabolic syndrome. Metformin is not a first-line treatment for infertility in PCOS patients. It is, however, an additional medication that, when combined with exercise and diet, improves menstrual cycle irregularity.

More research is needed to fully understand metformin efficacy and safety in pregnant women with PCOS. Metformin and lifestyle changes could be considered in adolescents with PCOS and adult PCOS women to treat weight, hormonal, and metabolic outcomes, according to recent international evidence-based guidelines. While metformin is considered safe for therapeutic use, some recent epidemiological studies have found phenotypic differences after prenatal exposure to metformin (Almalki et al., 2020).

Inositol, a second messenger that has an insulin-like effect on metabolic enzymes, is being investigated as a potential novel insulinsensitizing agent in PCOS women (Morley et al., 2017). According to a recent study, oral administration of Myo-inositol, alone or in combination with D-chiro-inositol, can recover unexpected ovulation and improve fertility in PCOS women (Macut, Bjekić-Macut, et al., 2017). However, general conclusions about the use of inositol remain elusive. Exenatide and liraglutide, two glucagon-like peptide 1 analogues, when combined with metformin, improved metabolic parameters and resulted in additional weight loss in obese PCOS patients (Jeanes \& Reeves, 2017). However, repeated studies and randomised clinical trials that confirm the alleged clinical efficacy of the drug classes mentioned earlier in women with PCOS are still lacking. Myo- 
inositol (MYO) consumption has been shown to improve ovulation and response to assisted fertilization in otherwise infertile women with PCOS (Altinok et al., 2018). A recent systematic review of the impact of MYO supplements in women with PCOS concluded that MYO supplements improved the hormonal and reproductive problems associated with PCOS (Sharpe et al., 2019). It also improved follicular development and oocyte maturation. MYO supplements are generally well tolerated at the currently recommended doses of 2-4 g/ day with minimal side effects; thus, its clinical application in PCOS management is important to consider (Q. Y. Wang et al., 2016).

Simvastatin therapy is another option for women with PCOS. Simvastatin (20 mg) in combination with an oral contraceptive pill (OCP) or an OCP alone were given to 48 PCOS patients (Abdalla et al., 2020). Using statins for treatment Serum testosterone levels were reduced, as were $\mathrm{LH}$ and the LH: FSH ratio, and lipid profiles were improved (Jensterle et al., 2020). Simvastatin and metformin both improved menstrual cyclicity and decreased hirsutism, acne, and ovarian volume (by up to 14\%), with simvastatin having the greatesteffect (Jeanes \& Reeves, 2017). There is substantial evidence that statins can assist with PCOS treatment. Statins (atorvastatin ,fluvastatin, pravastatin, rosuvastatin, and simvastatin) inhibit 3-hydroxy-3-methylglutaryl coenzyme A (HMG-CoA) reductase, a rate-controlling enzyme in the cholesterol biosynthesis pathway (Costello et al., 2019). By inhibiting this enzyme, the conversion of HMG-CoA to mevalonate is halted, and thus cholesterol synthesis is halted. In a randomized placebocontrolled trial, atorvastatin significantly reduced insulin resistance, inflammatory markers, and hyperandrogenaemia in PCOS women compared to placebo (A. Wang et al., 2019). In a further 12 weeks, metformin followup both atorvastatin and placebo demonstrated significant improvements in HOMA-IR, the free androgen index (FAI), total testosterone and sex hormone-binding globuline (SEHBG), in atorvastatin pre-treated group compared to a placebo group when atorvastatin and placebo were followed up for a further 12 weeks (Moran et al., 2020).

A novel and promising concept is another treatment including resveratrol (3,5,4-trihydroxy-trans-stilbene).

Some possible etiologies of PCOS, including hyperandrogenism, resistance to insulin and systemic chronic inflammation, lead us to consider PCOS therapy for resveratrol (Jin \& Xie, 2018). Resveratrol - a natural polyphenol found in grapes and fruit, is produced in plants as a natural defense mechanism in response to ultraviolet irradiation, fungal and bacterial infections (Jensterle et al., 2020). The potential of Resversatrol treatment for type 2 or PCOS diabetes and selected endocrinopathies is therefore new. Several studies in the field of resvereatrol are currently designed to improve brain blood flow, memory and concentration in patients who have IVF and to prevent OSHS in pre-diabetic conditions, chronic cardiac congestive disease, and lymphangioleyomatosis in patients with IVF (excluding the PCOS) (Della Corte et al., 2020). In IVF patients only $2000 \mathrm{mg}$ of resveratrol was used in the OHSS prevention study, only for nine days. These in vitro and in vivo data show the potential benefit of PCOS women's resveratrol therapy (Li et al., 2020).

\section{CONCLUSION}

Research is under way into possible medicines to enhance endocrinological, reproductive and cardiometabolic abnormalities in PCOS. There is occasionally a lot of new ideas for causative therapy for women with PCOS, and some of them are subject to extensive in vitro and in vivo research followed by clinical studies. Metformin - a sensitizer of insulin - has the most extensive PCOS history and is recommended as a firstline treatment to improve glycemic/metabolic abnormalities. Metformin is also proposed to restore menstrual regularity and enhance hyperandrogenemia as primary therapy in teenagers with PCOS. An improvement of clinical pregnancy and ovulation suggests that the use of metformin in the ovulation in obese females with PCOS remains preferable to clomiphene citrate. Statins promise to reduce Hyperandrogenemia, chronic inflammation and improve lipid profiles, as well as adjunctives. Statin and metformin combination may be an important future strategy for treatment. The latest therapeutic additional agent is resveratrol in PCOS with strong antiinflammatory, cardiovascular and antioxidants actions. 


\section{CONFLICT OF INTEREST}

The author declared that no competing interests.

\section{REFERENCES}

Abdalla, M. A., Deshmukh, H., Atkin, S., \& Sathyapalan, T. (2020). A review of therapeutic options for managing the metabolic aspects of polycystic ovary syndrome. Therapeutic Advances in Endocrinology and Metabolism, 11, 1-19. https://doi. org/10.1177/2042018820938305

Almalki, H. H., Alshibani, T. M., Alhifany, A. A., \& Almohammed, O. A. (2020). Comparative efficacy of statins, metformin, spironolactone and combined oral contraceptives in reducing testosterone levels in women with polycystic ovary syndrome: A network meta-analysis of randomized clinical trials. BMC Women's Health, 20(1), 1-6. https://doi. org/10.1186/s12905-020-00919-5

Altinok, M. L., Ravn, P., Andersen, M., \& Glintborg, D. (2018). Effect of 12-month treatment with metformin and/or oral contraceptives on health-related quality of life in polycystic ovary syndrome. Gynecological Endocrinology, 34(10), 859-863. https://doi.org/10.1080/095 13590.2018.1460343

Balen, A. H., Morley, L. C., Misso, M., Franks, S., Legro, R. S., Wijeyaratne, C. N., StenerVictorin, E., Fauser, B. C. J. M., Norman, R. J., \& Teede, H. (2016). The management of anovulatory infertility in women with polycystic ovary syndrome: An analysis of the evidence to support the development of global WHO guidance. Human Reproduction Update, 22(6), 687-708. https://doi.org/10.1093/ humupd/dmw025

Banaszewska, B., Pawelczyk, L., \& Spaczynski, R. (2019). Current and future aspects of several adjunctive treatment strategies in polycystic ovary syndrome. Reproductive Biology, 19(4), 309-315. https://doi. org/10.1016/j.repbio.2019.09.006

Brennan, L., Teede, H., Skouteris, H., Linardon, J., Hill, B., \& Moran, L. (2017). Lifestyle and Behavioral Management of Polycystic
Ovary Syndrome. Journal of Women's Health. https://doi.org/10.1089/ jwh.2016.5792

Charifson, M. A., \& Trumble, B. C. (2019). Evolutionary origins of polycystic ovary syndrome: An environmental mismatch disorder. Evolution, Medicine and Public Health, 2019(1), 50-63. https://doi. org/10.1093/emph/eoz011

Costello, M. F., Misso, M. L., Balen, A., Boyle, J., Devoto, L., Garad, R. M., Hart, R., Johnson, L., Jordan, C., Legro, R. S., Norman, R. J., Moran, L., Mocanu, E., Qiao, J., Rodgers, R. J., Rombauts, L., Tassone, E. C., Thangaratinam, S., Vanky, E., \& Teede, H. J. (2019). A brief update on the evidence supporting the treatment of infertility in polycystic ovary syndrome. Australian and New Zealand Journal of Obstetrics and Gynaecology, 59(6), 867-873. https://doi.org/10.1111/ajo.13051

Cozzolino, M., Vitagliano, A., Pellegrini, L., Chiurazzi, M., Andriasani, A., Ambrosini, G., \& Garrido, N. (2020). Therapy with probiotics and synbiotics for polycystic ovarian syndrome: a systematic review and meta-analysis. European Journal of Nutrition, 0123456789. https://doi. org/10.1007/s00394-020-02233-0

De Diego, M. V., Gómez-Pardo, O., Groar, J. K., López-Escobar,A.,Martín-Estal,I.,CastillaCortázar, I., \& Rodríguez-Zambrano, M. Á. (2020). Metabolic impact of current therapeutic strategies in Polycystic Ovary Syndrome: a preliminary study. Archives of Gynecology and Obstetrics, 0123456789. https://doi.org/10.1007/ s00404-020-05696-y

Della Corte, L., Foreste, V., Barra, F., Gustavino, C., Alessandri, F., Centurioni, M. G., Ferrero, S., Bifulco, G., \& Giampaolino, P. (2020). Current and experimental drug therapy for the treatment of polycystic ovarian syndrome. Expert Opinion on Investigational Drugs, 0(0). https://doi. org/10.1080/13543784.2020.1781815

Dokras, A., Playford, M., Kris-Etherton, P. M., Kunselman, A. R., Stetter, C. M., Williams, N. I., Gnatuk, C. L., Estes, S. J., Sarwer, D. B., Allison, K. C., Coutifaris, C., Mehta, N., \& Legro, R. S. (2017). Impact of hormonal contraception and weight loss on highdensity lipoprotein cholesterol efflux 
and lipoprotein particles in women with polycystic ovary syndrome. Clinical Endocrinology, 86(5), 739-746. https:// doi.org/10.1111/cen.13310

Jeanes, Y. M., \& Reeves, S. (2017). Metabolic consequences of obesity and insulin resistance in polycystic ovary syndrome: Diagnostic and methodological challenges. In Nutrition Research Reviews. https://doi.org/10.1017/ S0954422416000287

Jensterle, M., Kravos, N. A., Ferjan, S., Goricar, K., Dolzan, V., \& Janez, A. (2020). Long-term efficacy of metformin in overweightobese PCOS: Longitudinal follow-up of retrospective cohort. Endocrine Connections, 9(1), 44-54. https://doi. org/10.1530/EC-19-0449

Jin, P., \& Xie, Y. (2018). Treatment strategies for women with polycystic ovary syndrome. Gynecological Endocrinology, 34(4), 272-277. https://doi.org/10.1080/095 13590.2017.1395841

Kim, C. H., Chon, S. J., \& Lee, S. H. (2020). Effects of lifestyle modification in polycystic ovary syndrome compared to metformin only or metformin addition: A systematic review and meta-analysis. Scientific Reports, 10(1), 1-14. https://doi. org/10.1038/s41598-020-64776-w

Li, Y., Tan, J., Wang, Q., Duan, C., Hu, Y., \& Huang, W. (2020). Comparing the individual effects of metformin and rosiglitazone and their combination in obese women with polycystic ovary syndrome: a randomized controlled trial. Fertility and Sterility, 113(1), 197-204. https://doi. org/10.1016/j.fertnstert.2019.09.011

Macut, D., Bjekić-Macut, J., Rahelić, D., \& Doknić, M. (2017). Insulin and the polycystic ovary syndrome. Diabetes Research and Clinical Practice, 130, 163-170. https:// doi.org/10.1016/j.diabres.2017.06.011

Macut, D., Božić-Antić, I., Bjekić-Macut, J., \& Tziomalos, K. (2017). Management of endocrine disease: Polycystic ovary syndrome and nonalcoholic fatty liver disease. European Journal of Endocrinology, 177(3), R145-R158. https://doi.org/10.1530/EJE-16-1063

Moradi, F., Ghadiri-Anari, A., Dehghani, A., Vaziri, S. R., \& Enjezab, B. (2020). The effectiveness of counseling based on acceptance and commitment therapy on body image and self-esteem in polycystic ovary syndrome: An RCT. International Journal of Reproductive BioMedicine, 18(4), 243-252. https:// doi.org/10.18502/ijrm.v13i4.6887

Moran, L. J., Tassone, E. C., Boyle, J., Brennan, L., Harrison, C. L., Hirschberg, A. L., Lim, S., Marsh, K., Misso, M. L., Redman, L., Thondan, M., Wijeyaratne, C., Garad, R., Stepto, N.K., \& Teede,H.J.(2020).Evidence summaries and recommendations from the international evidencebased guideline for the assessment and management of polycystic ovary syndrome: Lifestyle management. Obesity Reviews, 21(10), 1-15. https:// doi.org/10.1111/obr.13046

Morgante, G., Massaro, M. G., Scolaro, V., Cappelli, V., Luddi, A., Troìa, L., \& De Leo, V. (2020). Metformin doses and body mass index: clinical outcomes in insulin resistant polycystic ovary syndrome women. European Review for Medical and Pharmacological Sciences, 24(15), 8136-8142. https://doi.org/10.26355/ eurrev_202008_22500

Morley, L. C., Tang, T., Yasmin, E., Norman, R. J., \& Balen, A. H. (2017). Insulinsensitising drugs (metformin, rosiglitazone, pioglitazone, D-chiroinositol) for women with polycystic ovary syndrome, oligo amenorrhoea and subfertility. Cochrane Database of Systematic Reviews, 2017(11). https:// doi.org/10.1002/14651858.CD003053. pub6

Podfigurna, A., Meczekalski, B., Petraglia, F., \& Luisi, S. (2020). Clinical, hormonal and metabolic parameters in women with PCOS with different combined oral contraceptives (containing chlormadinone acetate versus drospirenone). Journal of Endocrinological Investigation, 43(4), 483-492. https://doi.org/10.1007/ s40618-019-01133-3

Sharpe, A., Morley, L. C., Tang, T., Norman, R. J., \& Balen, A. H. (2019). Metformin for ovulation induction (excluding gonadotrophins) in women with polycystic ovary syndrome. Cochrane Database of Systematic 
Reviews, 2019(12). https://doi. org/10.1002/14651858.CD013505

Wang, A., Mo, T., Li, Q., Shen, C., \& Liu, M. (2019). The effectiveness of metformin, oral contraceptives, and lifestyle modification in improving the metabolism of overweight women with polycystic ovary syndrome: a network meta-analysis. Endocrine, 64(2), 220-232. https://doi. org/10.1007/s12020-019-01860-w

Wang, Q. Y., Song, Y., Huang, W., Xiao, L., Wang, Q. S., \& Feng, G. M. (2016). Comparison of drospirenone-with cyproterone acetate-containing oral contraceptives, combined with metformin and lifestyle modifications in women with polycystic ovary syndrome and metabolic disorders:
A prospective randomized control trial. Chinese Medical Journal, 129(8), 883890. https://doi.org/10.4103/03666999.179783

Zeng, X., Xie, Y. jie, Liu, Y. ting, Long, S. lian, \& Mo, Z. cheng. (2020). Polycystic ovarian syndrome: Correlation between hyperandrogenism, insulin resistance and obesity. Clinica Chimica Acta, 502, 214-221. https://doi.org/10.1016/j. cca.2019.11.003

Zhang, B., Zhang, B., Zhang, B., Zhang, B., Zhou, W., Zhou, W., Zhou, W., Zhou, W., Shi, Y., Shi, Y., Shi, Y., Shi, Y., Zhang, J., Cui, L., Cui, L., Cui, L., Cui, L., Chen, Z. J., Chen, Z. J., ... Chen, Z. J. (2020). Lifestyle and environmental contributions to ovulatory dysfunction 